\title{
Effect of Polyhydroxyl Compounds on the Thermal Stability and Structure of Laccase
}

\author{
Chen Kang, ${ }^{1,2}$ Dajun Ren ${ }^{1,2 *}$, Shuqin Zhang ${ }^{1,2}$, Xiaoqin Zhang1,2, Xiaoyong He ${ }^{1,2}$, \\ Zhiqun Deng ${ }^{1,2}$, Chaofan Huang ${ }^{1,2}$, Huiwen Guo ${ }^{1,2}$ \\ ${ }^{1}$ College of Resource and Environmental Engineering, Wuhan University of Science and Technology, Wuhan, China \\ ${ }^{2}$ Hubei Key Laboratory for Efficient Utilization and Agglomeration of Metallurgic Mineral Resources, \\ Wuhan University of Science and Technology, Wuhan, Hubei, China
}

Received: 18 June 2018

Accepted: 4 September 2018

\begin{abstract}
In this study, glucose, inositol, glycerol, and sorbitol have been chosen as chemical modifiers to enhance laccase stability. The regularity of laccase activity variations was studied at different temperatures. Low concentrations of four polyhydroxyl compounds can improve the thermal stability of laccase at the whole temperature gradient $\left(20 \sim 60^{\circ} \mathrm{C}\right)$. The effect of glycerol is best in the all additives, and the relative enzyme activity of laccase increased 1.35 times by glycerol, in the concentration of $0.6 \mathrm{~mol} / \mathrm{L}$. The conformation of modified laccase is characterized by UV, fluorescence and circular dichroism. The result shows that the polyhydroxy compounds can change the conformation of laccase by increasing the hydrogen bonds between the laccase molecules and prevent the adverse change due to the increase of temperature in the secondary structure of laccase. Therefore, low concentrations of polyhydroxy compounds could be served as an additive to improve the thermal stability of the laccase.
\end{abstract}

Keywords: polyhydroxyl compounds, laccase, thermal stability

\section{Introduction}

Laccases are copper-blue polyphenol oxidases that could utilize molecular oxygen to catalyze the oxidation reaction by a radical catalyzed reaction $[1$, 2]. As a catalyst, lactase has many advantages such as extensiveness of substrates, no secondary pollution and high efficiency [3, 4]. Laccases have many potential applications in the field of environmental remediation. However, natural laccases have many limitations such as poor enzyme activity stability and low renaturation

*e-mail: dj_ren@163.com ratio (the ratio of laccase activity after renaturation to laccase activity of the natural state). The actual application of laccase is limited when $\mathrm{pH}$ value and temperature exceed the suitable range $[5,6]$. Then chemical modification is often used to modify enzyme molecules to improve enzyme stability $[7,8]$. A chemical modification method of an enzyme is to modify it at the molecular level, which is that using chemical methods to combine macromolecules to some functional groups of the enzyme molecules to improve the biological properties of the enzyme [9]. Simon et al. found that glycerol, polyethylene glycol and sorbitol could maintain the activity of $\alpha$-chymotrypsin in high-concentration ethyl alcohol [10]. Martina et al. found that inositol and glucose could improve the protein conformation of 
glutamine synthetase and malate dehydrogenase, and increase their thermal stability [11]. This paper provides a feasible solution for improving the thermal stability of laccase by studying the effect of polyhydroxy compounds on laccase.

In this paper, sorbitol, glycerol, inositol, and glucose were selected as additives to study the regularity of variation of laccase activity at different temperatures and different additive concentrations. The structure of laccase was analyzed by ultraviolet spectrometer, fluorescence spectrometer and circular dichroism spectrometer to research the laccase enzymatic response mechanism under the protection of polyhydroxy compounds.

\section{Material and Methods}

\section{Solution Preparation and Determination}

Laccase (Trametes Versicolor) and 2, 2-azino-bis(3-ethylbenzothiazo line-6-sulfonic acid) (ABTS) were purchased from Sigma Company. The other chemical reagents were prepared in China and analytically pure. The buffer solution $\left(\mathrm{NaH}_{2} \mathrm{PO}_{4}-\mathrm{Na}_{2} \mathrm{HPO}_{4}\right)$ with $\mathrm{pH}$ value 3 were mixed with laccase to produce a laccase solution whose concentration is $0.2 \mathrm{U} / \mathrm{mL}$. Four kinds of polyhydroxy compounds (sorbitol, glycerol, inositol and glucose) were respectively dissolved in a buffer solution to obtain polyhydroxy compound solution at concentrations of $0,0.1,0.2,0.4,0.8$, and $1.2 \mathrm{~mol} / \mathrm{L}$. We mixed the polyhydroxy compounds solution with the laccase solution in an equal volume and heated in a constant temperature water bath to determine the relative enzyme activity of the laccase in the mixture at $20-70^{\circ} \mathrm{C}$. Fluorescence spectra, circular dichroism spectra and ultraviolet spectra of laccase solutions were determined using a fluorescence spectrometer (FP-6500, Jasco, Japan), circular dichroism spectrometer (J-810, Jasco, Japan) and ultraviolet spectrometer (UV-2550, Tsushima, Japan), respectively.

\section{Determining Laccase Activity and Thermodynamic Inactivation Curve}

A laccase solution of $\mathrm{pH}=3$ was put into the constant temperature water bath, then the laccase enzyme activity was determined every 20 minutes to make the thermodynamic inactivation curve of laccase.
The ABTS method was used to determine laccase activity: $2 \mathrm{~mL}$ enzyme solution and $1 \mathrm{~mL} 0.5 \mathrm{mmol} / \mathrm{L}$ ABTS was added to a cuvette to start reaction at room temperature, then determine the increase of the absorbance at $420 \mathrm{~nm}$ in the UV spectrophotometer in the first 3 minutes of the reaction. The OD value changes by 0.001 per minute, as an enzyme activity unit denoted as U.

If the inactivation laccase is in accordance with the first-order kinetics, then:

$$
\begin{aligned}
\ln \frac{A_{t}}{A_{0}} & =-k t \\
t_{1 / 2} & =\frac{\ln 2}{k}
\end{aligned}
$$

...where $\mathrm{T}$ is heating time, $\mathrm{A}_{\mathrm{t}}$ is laccase activity at the heating time $t, A_{0}$ is initial enzyme activity of laccase, $k$ is deactivation rate parameter, and $T_{1 / 2}$ is the half-life of laccase.

The Gibbs free energy formula for laccase at the same temperature is:

$$
\Delta \mathrm{G}=-\mathrm{RT} \ln \left(\frac{\mathrm{kh}}{\mathrm{k}_{\mathrm{b}} \mathrm{T}}\right)
$$

...where $\mathrm{R}$ is the molar gas constant, $\mathrm{T}$ is the thermodynamic temperature, $\mathrm{h}$ is the Planck constant, and $\mathrm{K}_{\mathrm{b}}$ is the Boltzmann constant.

\section{Results and Discussion}

\section{The Effect of Polyhydroxy Compounds Addition on Thermal Stability of Laccase}

The thermodynamic inactivation constant $\mathrm{k}$, halflife $\mathrm{t} 1 / 2$, and thermal inactivation free energy $\Delta \mathrm{G}$ were calculated (Table 1), according to Fig. 1 and the rendering method of thermodynamic inactivation curve. As seen in Table 1, the laccase activity decreased gradually with the passage of time and the increase in temperature. The result showed that laccase halflife was $144.41 \mathrm{~min}$ at $20^{\circ} \mathrm{C}$ and $62.45 \mathrm{~min}$ at $40^{\circ} \mathrm{C}$, and the half-life of the laccase reached a minimum of 18.84 minutes at $60^{\circ} \mathrm{C}$. Fig. 2 showed that the introduction of polyhydroxyl compounds could effectively extend the half-life of laccase $\mathrm{t} 1 / 2$ at an ambient temperature of $40^{\circ} \mathrm{C}$.

Table 1. Laccase thermodynamic parameters.

\begin{tabular}{|c|c|c|c|c|c|}
\hline $\mathrm{T} /{ }^{\circ} \mathrm{C}$ & 20 & 30 & 40 & 50 & 60 \\
\hline $\mathrm{k} / \mathrm{min}^{-1}$ & 0.0048 & 0.0067 & 0.0111 & 0.0186 & 0.0368 \\
\hline $\mathrm{t}_{1 / 2} / \mathrm{min}$ & 144.41 & 103.45 & 62.45 & 37.27 & 18.84 \\
\hline$\Delta \mathrm{G} /(\mathrm{KJ} / \mathrm{mol})$ & 94.74 & 97.22 & 99.2 & 101.06 & 102.38 \\
\hline
\end{tabular}




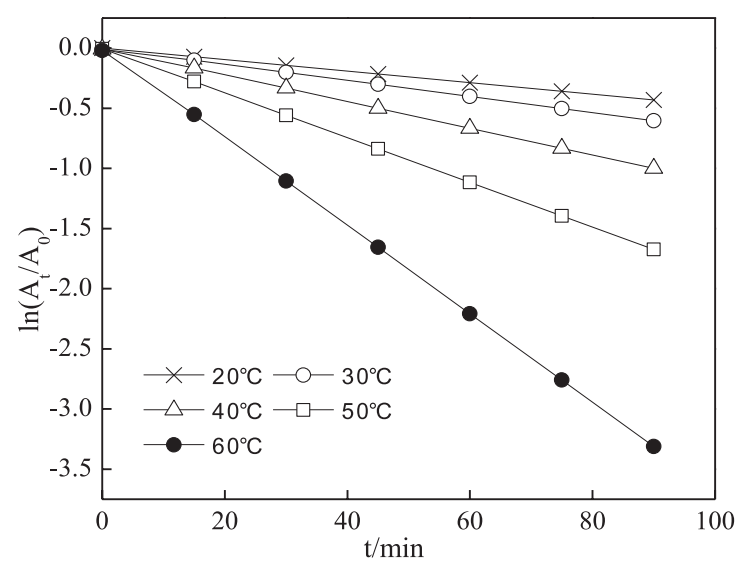

Fig. 1. Thermodynamic inactivation curve of laccase.

\section{The Effect of Additives on Stability of Enzyme Activities at Different Temperatures}

As shown in Fig. 3, with the increasing concentrations of glucose and inositol, laccase activity increased at first and then decreased during $20-50^{\circ} \mathrm{C}$ (Fig. 3a-d). The laccase activity peaked when the glucose and inositol groups were respectively at concentrations of $0.1 \mathrm{~mol} / \mathrm{L}$ and $0.2 \mathrm{~mol} / \mathrm{L}$, but a high concentration of glucose inhibited laccase activity. In the glycerol and sorbitol groups, the activity of laccase was positively correlated with the additive concentration in the range of $20-50^{\circ} \mathrm{C}$ (Fig. 3a-d). Laccase activity was the maximum when the concentration of polyhydroxyl compounds was $0.6 \mathrm{~mol} / \mathrm{L}$, and relative enzyme activity increased by 1.17 and 1.37 times, respectively. However, the protective effect of additives on laccase was significantly reduced when the temperature exceeded $60^{\circ} \mathrm{C}$. The conformation of laccase was completely destroyed by the excessive temperature, which became the main influence factor of laccase activity. In general, the choice of concentration and type of polyhydroxyl

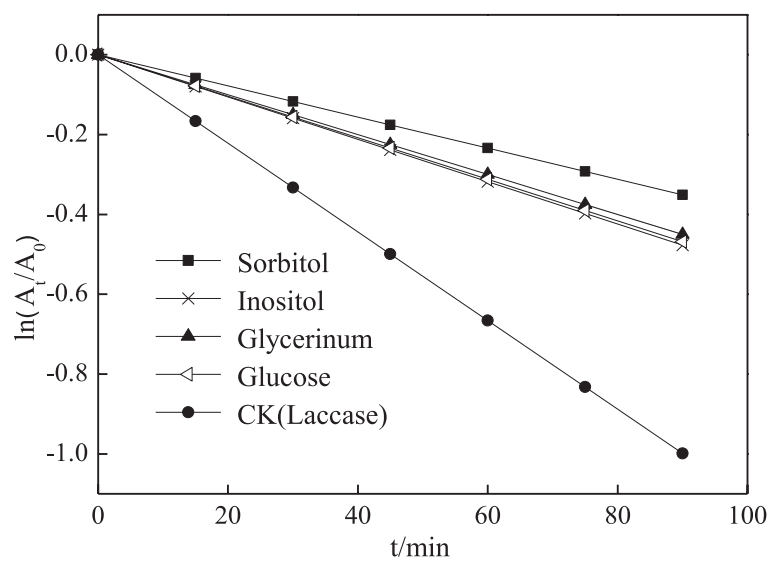

Fig. 2. Thermodynamic inactivation curve of laccase after adding polyhydroxy compounds. a)

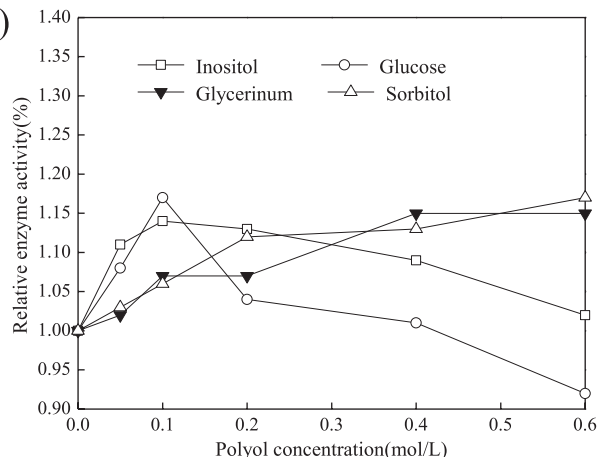

b)
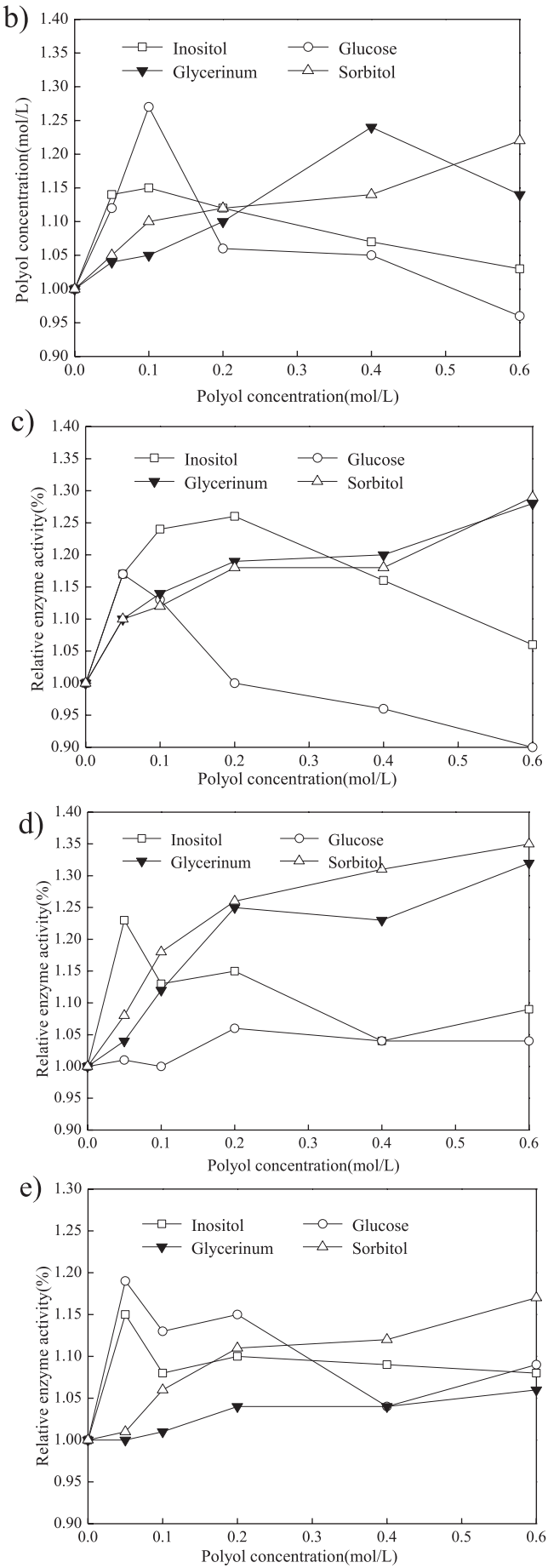

Fig. 3. Effect of polyhydroxy compounds on laccase activity at different temperatures: a) $20^{\circ} \mathrm{C}$, b) $30^{\circ} \mathrm{C}$, c) $40^{\circ} \mathrm{C}$, d) $50^{\circ} \mathrm{C}$, e) $60^{\circ} \mathrm{C}$. 


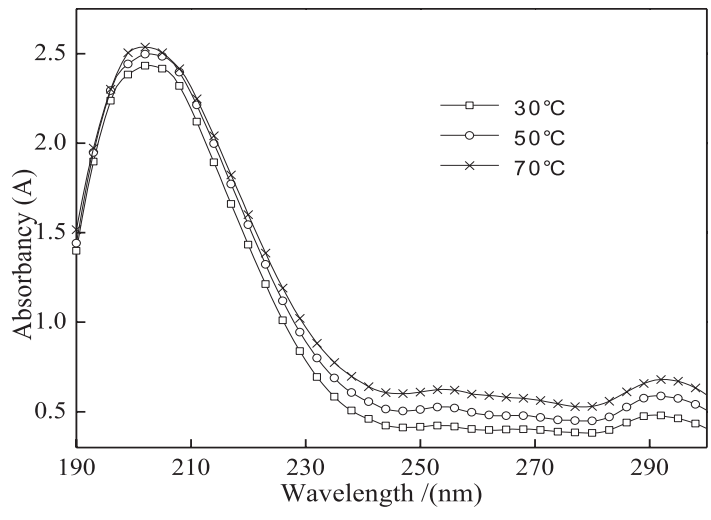

Fig. 4. Ultraviolet spectra of laccase at different temperatures.

compounds had an important influence on the thermal stability of laccase. $0.6 \mathrm{~mol} / \mathrm{L}$ sorbitol was the best for laccase protection in all temperature gradients. When the glucose and inositol concentration exceeds the optimal concentration, the effect on the improvement of laccase activity began to weaken, resulting in a decrease in the renaturation ratio of the laccase. Therefore, the low concentration of polyhydroxyl compound has the best effect on improving the temperature stability of laccase in a highly hydrophilic polyol solution.

\section{The Ultraviolet Spectra Analysis of Laccase}

As shown in Fig. 4, with the ambient temperature gradually increasing, the absorption peak of the peptide bond was enhanced gradually at around $200 \mathrm{~nm}$. This indicated that laccase molecules would be unfolded and alter the specific spatial arrangement of the peptide chains with the increasing temperature, then affect laccase activity. The characteristic absorption peak of laccase at $290 \mathrm{~nm}$ was caused by the combination of phenylalanine, tryptophan and 13 tyrosines. With the solution temperature increasing, the absorption peak of laccase at $290 \mathrm{~nm}$ gradually increased. There was a strong possibility that amino acid residues were exposed

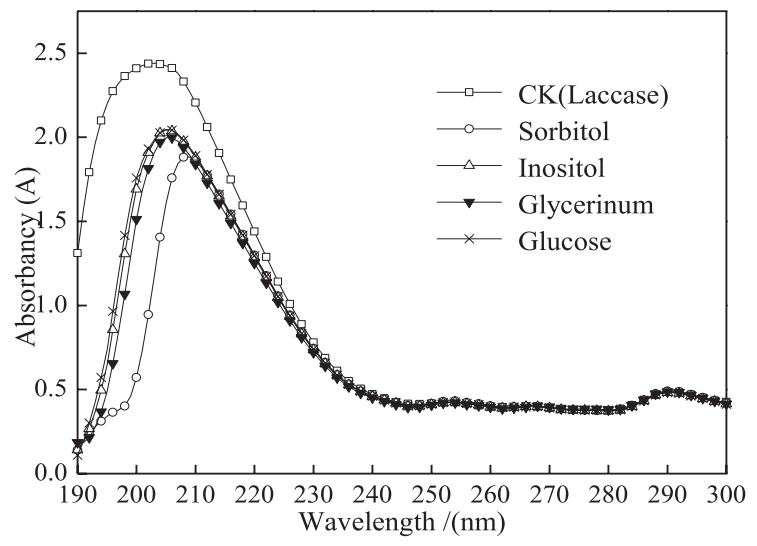

Fig. 5. Ultraviolet spectrum of laccase after adding polyhydroxy compounds.

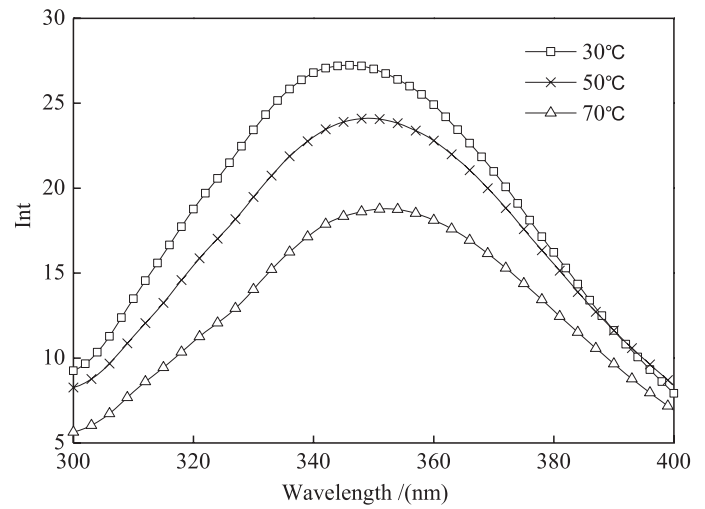

Fig. 6. Fluorescence spectra of laccase at different temperatures.

from the inside of the hydrophobic surface by heating, thus affecting the activity of laccase. As illustrated in Fig. 5, the absorption peak of the peptide bond at 190 $220 \mathrm{~nm}$ after the addition of the polyhydroxy compounds was significantly decreased, and the absorption peak showed a slight redshift. This showed that the conformation of the laccase in the solution changed after the addition of polyhydroxy compounds. With reference to Fig. 4, it could be seen that the intensity of the absorption peak of the peptide bond of the laccase increased with the rising temperature, indicating that extension of the laccase peptide chain was induced with the increase of temperature. The absorption peak of the peptide bond became weaker after the addition of the polyhydroxy compounds, indicating that the interaction of the polyhydroxy compounds with laccase attenuated the adverse effects of elevated temperatures on the laccase structure. There was an ultraviolet absorption peak of phenylalanine, tryptophan and tyrosine at 280-300 $\mathrm{nm}[12,13]$. The absorption peak at this position reflected the change in the microenvironment of the laccases amino acid residue after the addition of polyhydroxy compounds. From Fig. 5, the intensity of the absorption peak of laccases changed slightly, and there was no blueshift and redshift. Since the change of the amino acid residue microenvironment could not be analyzed by UV spectroscopy, the tyrosine tryptophan residue was further analyzed by fluorescence spectroscopy.

\section{The Fluorescence Analysis of Laccase}

Acording to Fig. 6, the fluorescence intensity of tryptophan residues decreased with increasing temperature, indicating that heating will cause fluorescence quenching of tryptophan residues of laccase. Fluorescence quenching is the flipping of the laccase peptide bond caused by rising temperature, making the hydrophobic tryptophan residues buried, which leads to a decrease in fluorescence intensity. The appearance of fluorescence quenching indicated that the temperature change will affect the conformation of laccase. From Fig. 7, it could be seen that the fluorescence 


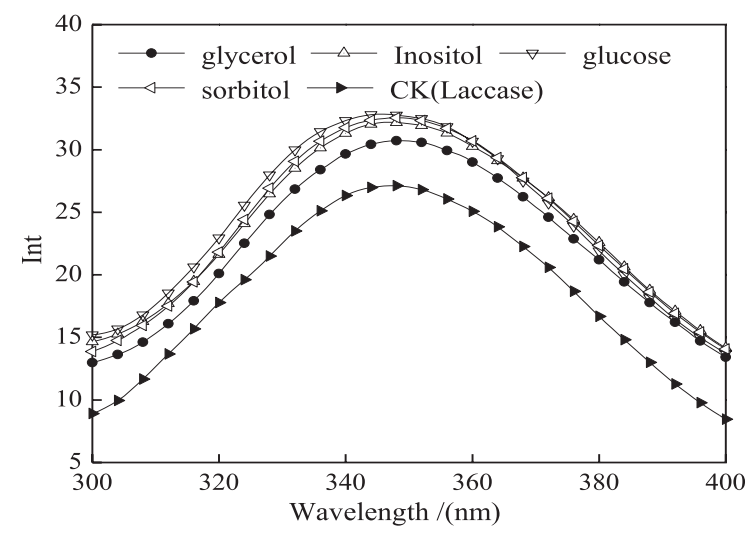

Fig. 7. Fluorescence spectra of laccase after adding polyhydroxy compounds.

spectrum was represented by the tryptophan residue of the laccases after the addition of the polyhydroxy compounds, in which the sorbitol fluorescence intensity of the sorbitol group was maximum. That it was consistent with the laccases activity showed that the interaction between sorbitol and laccase molecules was closer. The $\log \mathrm{P}$ (concentration of drug in octanol concentration of drug in aqueous solution) values of sorbitol, glycerol, inositol and glucose were -4.67, $-2.32,-2.11$ and -1.88 , respectively. The hydrophilicity of sorbitol was significantly greater than that of other alcohols. The hydroxyl groups were easier to separate from sorbitol, which helped to enhance hydrogen bonds between the enzyme protein molecules. After the polyhydroxy compounds were added to the solution, the fluorescence intensity of the tryptophan residue of laccase increased, which means that the polyhydroxy compounds could actually prevent the laccases from accumulating due to temperature. However, when the ambient temperature was $70^{\circ} \mathrm{C}$, the fluorescence intensity of laccase decreases sharply, indicating that the polyol had a limited protective effect on laccase. When the temperature covers the upper limit, the temperature will directly destroy the conformation of laccase, and the temperature became the main influence factor of laccase activity.

\section{The Circular Dichroism Analysis of Laccase}

Fig. 8 shows that the absorption spectrum of laccase changed slightly when the solution temperatures were $30^{\circ} \mathrm{C}$ and $50^{\circ} \mathrm{C}$, respectively. This indicated that the secondary structure of laccase was relatively stable. Combined with Fig. 3, laccase activities were different at large at $30^{\circ} \mathrm{C}$ and $50^{\circ} \mathrm{C}$, but the circular chromatogram demonstrated that the secondary structure of laccase didn't have a significant difference at these two temperatures. This indicated that the thermodynamic inactivation of laccase was reversible and the renaturation ratio was high. When the temperature was heated to $70^{\circ} \mathrm{C}$, the intensity of the absorption peak of

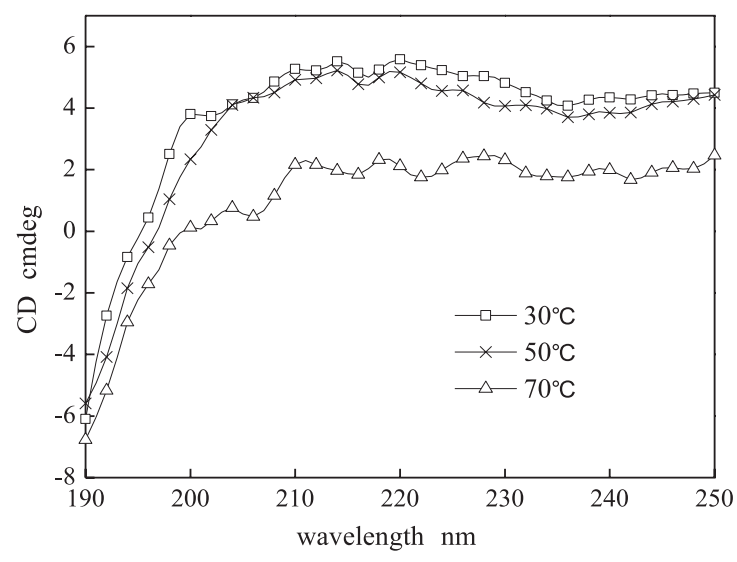

Fig. 8. Circular dichroism of laccase at different temperatures.

laccases was significantly reduced, and the laccases were completely inactivated, indicating that the secondary structure of laccases was directly destroyed at this time. As shown in Fig. 8, the secondary structure content of $\beta$-sheet on the laccases increased after the addition of polyhydroxy compounds, and the content of the random coil decreased. This indicated that the random coil in the secondary structure of the laccase was converted to the $\beta$-sheet after the addition of polyhydroxy compounds, thereby improving the thermal stability of laccase. Therefore, the thermal stability of the laccases had been improved.

\section{The Protection Mechanism of Polyhydroxy Compounds on Laccase Thermal Stability}

We analyzed the changes of laccases at different temperatures in UV, fluorescence, and circular dichroism spectra. During the temperature ranging $30-70^{\circ} \mathrm{C}$, the increase of the peptide bond absorption peak of laccase indicated that the increase of temperature would lead to the extension of laccase structure. Due to the increase of temperature, the structure of laccase would be

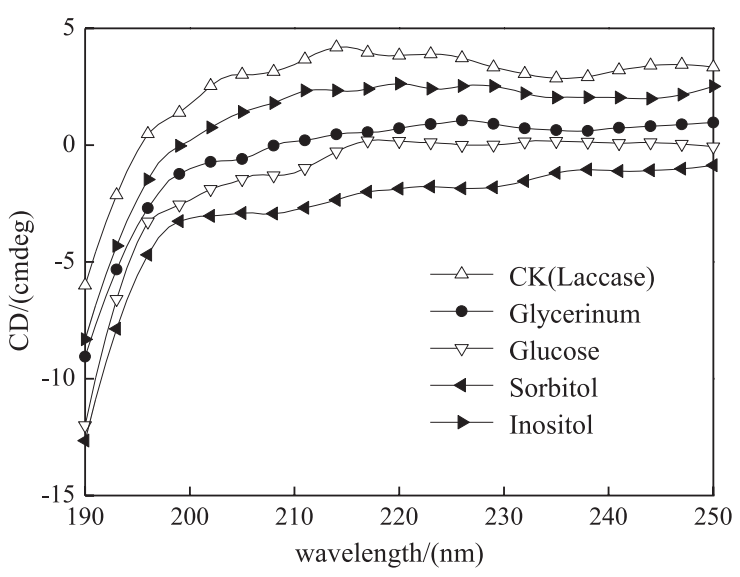

Fig. 9. Circular dichroism of laccase after adding polyhydroxy compounds. 
transformed from a tight, ordered conformation to a loose, random one. The fluorescence quenching occurred in tryptophan residues of laccase peptide bond when the ambient temperature arose to $70^{\circ} \mathrm{C}$. It was probable that the reduction of solution viscosity was caused by increasing temperature. The increase of kinetic energy of gas solutes and liquid solvents resulted in the increase of collision probability between fluorescence and other molecules. The excited state of fluorescence molecules released energy through molecular collision or intramolecular energy transferring. The excited fluorescence back to the ground state in the form of non-fluorescent emission resulted in fluorescence quenching.

The test results showed that the UV spectrum of the laccase was not much different after adding different polyhydroxy compounds. It was probable that the hydroxyl in the additives combined with the laccase molecule to create hydrogen bonds to enhance the thermal stability of laccase. Furthermore, each additive saturated the hydrogen bond of laccase, which reflected that the low concentration of polyhydroxyl compounds could improve the thermal stability of laccase [14]. The circular dichroism of laccase further indicated that the secondary structure of the laccase $\beta$-sheet was significantly increased [15]. The polyhydroxy compounds could interact with the laccases molecule, so that the random coil and $\beta$-sheet fold in the laccase molecule was maintained at a suitable ratio. At the same time, the polyhydroxy compounds could resist the adverse effects of temperature changes on the laccases molecule. However, laccase activity was inhibited in high concentration of glucose and inositol at $40^{\circ} \mathrm{C}$. The secondary structure of the $\beta$-sheet was significantly increased by excess alcohol hydroxyl, which resulted in the increase of molecular association, the formation of soluble polymers, and the reduced renaturation ratio of laccase $[16,17]$.

\section{Conclusions}

The thermodynamic inactivation of laccase coincides with the first-order reaction. With the temperature increasing, the half-life of laccase has been decreased. At the same time, the activity and conformation of lactase would be changed. However, the thermal stability of laccase will be significantly improved after the addition of polyhydroxy compounds. Laccase activity shows a trend of increasing and then decreasing with the increasing inositol and glucose concentrations during the concentration range of $0.1 \mathrm{~mol} / \mathrm{L} \sim 0.6 \mathrm{~mol} / \mathrm{L}$. However, laccase has been irreversibly inactivated when the ambient temperature exceeds $70^{\circ} \mathrm{C}$. The thermal stability of laccase could not be improved by polyhydroxy compounds.

According to UV spectra, circular dichroism and fluorescence spectra of laccase, polyhydroxy compounds forms hydrogen bonds between the secondary structure of laccase protein, and effectively prevent the secondary structure of laccase from changing with the increase of temperature. The increase of hydrogen bonds could maintain the stability of $\alpha$-helix and $\beta$-sheet. The polyhydroxy compounds not only formed hydrogen bonds with water molecules to increase the viscosity of the solution, but also to decrease the aggregation and sedimentation of laccase molecules caused by the increase of temperature.

\section{Acknowledgements}

This work was supported by the National Natural Science Foundation of China (grant No. 41571306), the Project of Excellent Fund in Hubei (grant No. 2018CFA067) and Hubei Key Laboratory for Efficient Utilization and Agglomeration of Metallurgic Mineral Resources (grant No. 2017zy003).

\section{Conflict of Interest}

The authors declare no conflict of interest.

\section{References}

1. GIARDINA P., FARACO V., PEZZELLA C., PISCITELLI A., VANHULLE S., SANNIA G. Laccases: a never-ending story [J]. Cellular \& Molecular Life Sciences, 67 (3), 369, 2010.

2. MORE S.S., RENUKA P.S., PRUTHVI K., SWETHA M., MALINI S., VEENA S.M. Isolation, Purification, and Characterization of Fungal Laccase fromPleurotussp [J]. Enzyme Research, 2011, (2011-08-09), 2011 (1), 248735, 2011.

3. STRONG P.J., CLAUS H. Laccase: A Review of Its Past and Its Future in Bioremediation [J]. Critical Reviews in Environmental Science \& Technology, 41 (4), 373, 2011.

4. LLORET L., EIBES G., L-CHAU T.A., MOREIRA M.T., FEIJOO G., LEMA J.M. Laccase-catalyzed degradation of anti-inflammatories and estrogens [J]. Biochemical Engineering Journal, 51 (3), 124, 2010.

5. LU C., CAO L., LIU R., LEI Y., DING G. Effect of common metal ions on the rate of degradation of 4-nitrophenol by a laccase- $\mathrm{Cu}^{2+}$ synergistic system [J]. Journal of Environmental Management, 113 (1), 1, 2012.

6. ZEEB B., GIBIS M., FISCHER L., WEISS J. Crosslinking of interfacial layers in multilayered oil-in-water emulsions using laccase: Characterization and $\mathrm{pH}$-stability $[\mathrm{J}]$. Food Hydrocolloids, 27 (1), 126, 2012.

7. BOUTUREIRA O., BERNARDES G.J. Advances in chemical protein modification [J]. Chemical Reviews, 115 (5), 2174, 2015.

8. RODRIGUES R.C., BERENGUER-MURCIA A., FERNANDEZ-LAFUENTE R. ChemInform Abstract: Coupling Chemical Modification and Immobilization to Improve the Catalytic Performance of Enzymes [J]. Advanced Synthesis \& Catalysis, 353 (13), 2216, 2011.

9. RODRIGUES R.C., BERENGUER-MURCIA Á., FERNANDEZ-LAFUENTE R. Coupling chemical 
modification and immobilization to improve the catalytic performance of enzymes [J]. Advanced Synthesis \& Catalysis, 353 (13), 2216, 2011.

10. SIMON L.M., KOTORM N.M., GARAB G., LACZK I. Effects of polyhydroxy compounds on the structure and activity of alpha-chymotrypsin [J]. Biochemical \& Biophysical Research Communications, 293 (1), 416, 2002.

11. ORTBAUER M., POPP M. Functional role of polyhydroxy compounds on protein structure and thermal stability studied by circular dichroism spectroscopy [J]. Plant Physiology \& Biochemistry, 46 (4), 428, 2008.

12. DEMCHENKO A.P. Ultraviolet spectroscopy of proteins [M]. Springer Science \& Business Media, 2013.

13. GHOSH S., BADRUDDOZA A., UDDIN M., HIDAJAT $\mathrm{K}$. Adsorption of chiral aromatic amino acids onto carboxymethyl- $\beta$-cyclodextrin bonded $\mathrm{Fe} 3 \mathrm{O} 4 / \mathrm{SiO} 2$ coreshell nanoparticles $[\mathrm{J}]$. Journal of colloid and interface science, 354 (2), 483, 2011.
14. RANI A., VENKATESU P. Insights into the interactions between enzyme and co-solvents: stability and activity of stem bromelain [J]. International Journal of Biological Macromolecules, 73 189, 2015.

15. LI X., XU L., WANG G., ZHANG H., YAN Y. Conformation studies on Burkholderia cenocepacia lipase via resolution of racemic 1-phenylethanol in nonaqueous medium and its process optimization [J]. Process Biochemistry, 48 (12), 1905, 2013.

16. LIU W., BRATKO D., PRAUSNITZ J.M., BLANCH H.W. Effect of alcohols on aqueous lysozyme-lysozyme interactions from static light-scattering measurements $[\mathrm{J}]$. Biophysical Chemistry, 107 (3), 289, 2004.

17. LI J.J., LIU Y.D., WANG F.W., MA G.H., SU Z.G. Hydrophobic interaction chromatography correctly refolding proteins assisted by glycerol and urea gradients [J]. Journal of Chromatography A, 1061 (2), 193, 2004. 